\title{
Evaluation of an algorithm ascertaining cases of osteonecrosis of the jaw in the Swedish National Patient Register
}

This article was published in the following Dove Press journal:

Clinical Epidemiology

3 January 2013

Number of times this article has been viewed

\author{
Johan Bergdahl' \\ Fredrik Jarnbring ${ }^{2}$ \\ Vera Ehrenstein ${ }^{3}$ \\ Henrik Gammelager ${ }^{3}$ \\ Fredrik Granath' \\ Helle Kieler' \\ Madeleine Svensson' \\ Grethe S Tell ${ }^{4}$ \\ Ylva Trolle Lagerros' \\ 'Centre for Pharmacoepidemiology, \\ Department of Medicine Solna, \\ Karolinska Institutet, Stockholm, \\ Sweden; ${ }^{2}$ Department of Oral \\ and Maxillofacial Surgery Solna, \\ Karolinska University Hospital, \\ Stockholm, Sweden; ${ }^{3}$ Department \\ of Clinical Epidemiology, Aarhus \\ University Hospital, Aarhus, Denmark; \\ ${ }^{4}$ Department of Public Health and \\ Primary Health Care, University of \\ Bergen, Bergen, Norway
}

Correspondence: Ylva Trolle Lagerros Unit of Clinical Epidemiology, Department of Medicine Solna, Building T2, Karolinska University Hospital, SE-17I 76 Stockholm, Sweden

Tel +46851779 I73

Fax +46 85I 779304

Email ylva.trolle@ki.se
Background: Osteonecrosis of the jaw (ONJ) is a medical condition associated with antiresorptive drugs, among others, used to treat osteoporosis and bone metastasis. Currently, there is no consensus regarding the definition of ONJ, and no ONJ-specific International Classification of Diseases-10 code exists. Therefore, register-based studies of this condition may be troublesome.

Purpose: To evaluate an algorithm ascertaining ONJ cases in an attempt to facilitate future assessments of ONJ in clinical and epidemiological studies.

Methods: By means of the Patient Register and the Prescribed Drug Register, we identified all postmenopausal female residents in Sweden from 2005 through 2009. To identify potential cases of ONJ, we employed an algorithm including the following conditions: periapical abscess with sinus, inflammatory conditions of jaws, alveolitis of jaws, idiopathic aseptic necrosis of bone, osteonecrosis due to drugs, osteonecrosis due to previous trauma, other secondary osteonecrosis, other osteonecrosis, and unspecified osteonecrosis. Women seen at departments of oral and maxillofacial surgery, with at least one of the conditions, were classified as potential cases of ONJ. Conditions in anatomic sites other than the jaw were excluded. Validation was performed through medical record review. Case confirmation was based on the ONJ definition by the American Association of Oral and Maxillofacial Surgeons. The algorithm was evaluated by positive predictive values (PPVs) stratified by diagnosis.

Results: For the 87 potential cases identified through our algorithm, the medical records were obtained for 83 . The overall PPV was $18 \%$ (95\% confidence interval (CI) $10 \%-28 \%$ ). The highest PPV was observed in osteonecrosis due to drugs (83\%, 95\% CI 36\%-100\%). Several diagnoses had a PPV of 0 or were not used at all (periapical abscess with sinus, alveolitis of jaws, idiopathic aseptic necrosis of bone, osteonecrosis due to previous trauma, other secondary osteonecrosis, other osteonecrosis, and unspecified osteonecrosis).

Conclusion: It was possible to ascertain cases of ONJ from the Swedish registers using this algorithm; however, the PPV was low. Thus, further refinements of the algorithm are necessary.

Keywords: bisphosphonate-associated osteonecrosis of the jaw, epidemiology, methods, ONJ, registries, validation

\section{Introduction}

Osteonecrosis of the jaw (ONJ), which should be separated from osteoradionecrosis of the jaw, was first described in $2003,{ }^{1}$ and has been increasingly reported in the past few years. Since the majority of all cases have been associated with treatment of bisphosphonates, it is often called bisphosphonate-related osteonecrosis of the jaw. The association of phosphorus compounds with necrosis of the jaw was observed for the first time during the 19th century. Among workers in the matchmaking industry, 
cases of a painful condition with infection and exposed bone in the mouth were noted. This novel condition was named phossy-jaw, and since antibiotics did not exist in those days, it was often lethal. Following the decreased use of white phosphorus and improved working conditions in the industry, phossy-jaw disappeared as a medical issue. ${ }^{2}$ The modern use of bisphosphonates, however, has led to the recurrence of a related condition, namely ONJ.

There is no international consensus regarding the definition of ONJ. ${ }^{3}$ Two widely used definitions are those suggested by the American Association of Oral and Maxillofacial Surgeons and the American Society of Bone and Mineral Research. ${ }^{4,5}$ These, and most other, proposed diagnostic criteria of ONJ require that the patient is, or has been, under treatment with bisphosphonates. ${ }^{6}$ Lately, however, several reports have been published concerning the development of ONJ during antiresorptive treatment with the monoclonal antibody denosumab. ${ }^{7-10}$ Thus, the bisphosphonate criteria may need to be revised.

Although ONJ may arise spontaneously, it usually develops as a result of trauma to the jaw bone, such as through dental extractions or jaw surgery. ${ }^{11}$ Clinically, ONJ is manifested as nonviable, exposed bone in the mouth. The area around the exposed bone is typically inflamed, and both the soft tissue and the bone itself often become infected. ${ }^{12,13}$ Sequestration of bone and intraoral fistula development may occur, ${ }^{13}$ and on $\mathrm{x}$-ray, radiolucency as well as radiopacities may be seen. ${ }^{14}$ Even though ONJ is usually painful, sometimes there are no other symptoms beyond the exposed bone. In severe cases, however, pathological fractures and extra oral fistulas may develop if the necrotic jaw bone becomes infected. ${ }^{13}$ Figure 1 shows an ONJ lesion in the maxilla.

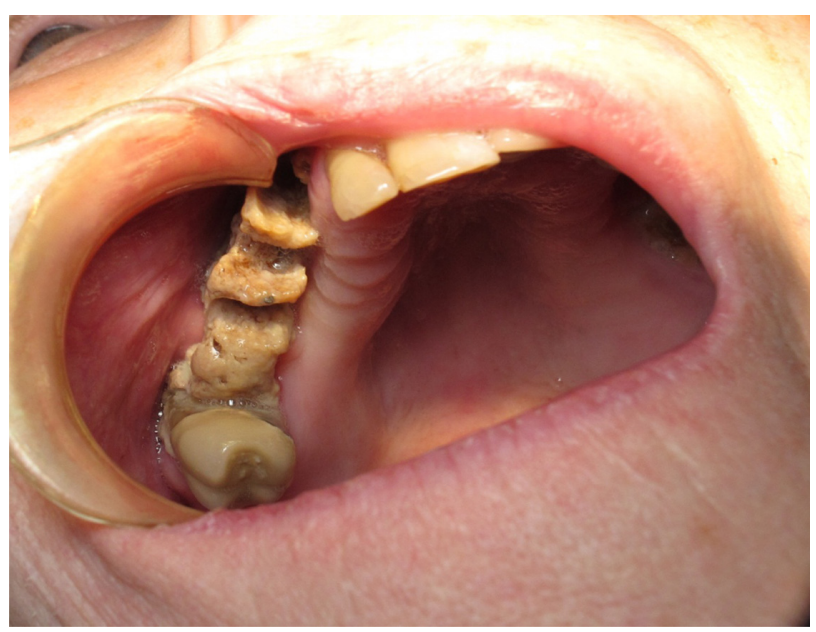

Figure I Patient with ONJ lesion in the right maxilla. Abbreviation: ONJ, osteonecrosis of the jaw.
Several risk factors have been associated with the development of ONJ. High cumulative doses of bisphosphonates and dentoalveolar surgery have been considered the strongest predictors of ONJ. ${ }^{15,16}$ Even though a definitive causal relationship between bisphosphonates and ONJ has still not been established, the most widely accepted explanations of the pathophysiology behind ONJ focus on different effects of bisphosphonates. ${ }^{17}$

Because ONJ is an uncommon condition, it is difficult to conduct studies reliably estimating the true magnitude of its risk. Performing studies based on large registers is one way to overcome these limitations in statistical power. On the other hand, besides the lack of a single accepted definition of ONJ, ${ }^{13}$ there is no ONJ specific ICD-10 (the International Classification of Diseases and Related Health Problems Tenth Revision) code and no consensus regarding which code to use instead. Hence, epidemiological studies of ONJ are troublesome, especially register-based ones, rendering assessments of risk factors, safety of treatment, and incidence data uncertain.

Despite the aforementioned difficulties conducting epidemiological studies on ONJ, register-based studies are likely to be one of the most suitable methods to study this condition. Therefore, in an attempt to facilitate future assessments of the epidemiology of $\mathrm{ONJ}$, we herein report on the evaluation of an algorithm ascertaining ONJ cases using data from the Swedish National Patient Register.

\section{Material and methods}

Postmenopausal osteoporotic women, who are highly likely to be prescribed bisphosphonates, were selected as study subjects. These patients were identified by means of the Swedish Patient Register and the Swedish Prescribed Drug Register. The Swedish Patient Register comprises all health care visits in inpatient care, and since 2001 all physician visits in specialized outpatient care as well. Both public and private health care providers are obligated to report these health care visits annually. ${ }^{18,19}$ Further, the Patient Register contains information on which hospital and department each diagnosis has been recorded, as well as the unique national registration number of each patient - a number given to all residents of Sweden. Thus, the national registration number allows for the linkage between the Patient Register and medical records on a patient level. Since 1997, diagnoses in the National Patient Register have been registered according to the Swedish version of the ICD-10. ${ }^{20}$ The Swedish Prescribed Drug Register contains data on all drugs dispensed by prescription. It includes information about the dispensed product, and the 
national registration number of the patient receiving the prescription. ${ }^{21}$

Postmenopausal women were defined as women aged 55 years or older during the study period. Among these women, osteoporosis was defined using an algorithm that required an osteoporosis code or an osteoporotic fracture code recorded in the Patient Register, or an osteoporotic medication code recorded in the Prescribed Drug Register.

To identify potential cases of ONJ, we employed an algorithm including the following conditions by their ICD-10 codes: periapical abscess with sinus (K04.6), inflammatory conditions of jaws (K10.2), alveolitis of jaws (K10.3), idiopathic aseptic necrosis of bone (M87.0), osteonecrosis due to drugs (M87.1), osteonecrosis due to previous trauma (M87.2), other secondary osteonecrosis (M87.3), other osteonecrosis (M87.8), and unspecified osteonecrosis (M87.9). Conditions in an anatomic site other than the jaw were excluded. Only women seen at departments of oral and maxillofacial surgery, from January 1, 2005 to December 31, 2009 , with a record of at least one of the diagnostic codes, were classified as potential cases of ONJ. We applied this restriction to avoid the inclusion of osteonecrosis localized outside the oral and maxillofacial region.

Validation of the potential cases of ONJ was performed by the author (JB) through medical record review. Patient identity was blinded; however, all other details in the medical record were available to the reviewer (for example ICD coding and caregiver). The medical records were identified using the information available in the Patient Register on national registration number, place of diagnosis, and date. For each potential case, the medical records of all visits to the department of oral and maxillofacial surgery coded with an ICD code included in our algorithm, and all related medical records, were obtained from the department in question. Case confirmation was based on the following criteria modified from the American Association of Oral and Maxillofacial Surgeons: ${ }^{4}$

1. A lesion in the oral cavity in the form of an exposed alveolar or palatal bone where gingiva or mucous membrane is usually found;

2. Absence of healing after 8 weeks despite adequate treatment; and

3. No prior radiation towards the head, face, or mouth.

Cases were classified as: (a) confirmed ONJ, or (b) not ONJ. All three criteria had to be fulfilled for confirmation.

The confirmed cases were characterized by age, comorbidities, medications, and ONJ specific characteristics such as localization of lesions and prior dentoalveolar surgery.
Comorbidities included a record of malignancy, diabetes mellitus, hypertension, rheumatoid arthritis, polymyalgia rheumatica, systemic lupus erythematosus, coagulopathia, renal failure, or hernia. History of medicine intake included bisphosphonates, cytostatics, glucocorticoids, antiangiogenetics, teriparatide, proton-pump inhibitors, and antihistamines. Characterization was based on medical records for all characteristics except malignancy, which was based on a combination of medical records and register data.

Positive predictive values (PPVs) and associated 95\% confidence intervals (CIs) were calculated for each diagnostic code and as a compound outcome, including all of the diagnostic codes. PPV was defined as the proportion of all potential cases fulfilling the criteria for ONJ in our medical record review (ie, PPV was calculated by dividing the number of confirmed cases by the number of potential cases).

To assess whether modifications of the algorithm would increase the PPV without decreasing the sensitivity, additional analyses were performed. All codes with a PPV of 0 were excluded from the algorithm. We also sharpened the criteria for becoming a potential case among cases coded with inflammatory conditions of jaws (K10.2). Thus, among these cases, only those coded with inflammatory conditions of jaws in two or more visits to the department of oral and maxillofacial surgery were considered potential cases. We also analyzed how PPV and sensitivity were affected when adding a requirement of a 6 -month interval between the two visits.

Sensitivity was defined as the proportion of all confirmed cases identified by the original algorithm that was identified by the modified algorithm. Thus, sensitivity was calculated by dividing the number of confirmed cases found with the modified algorithm by the number of confirmed cases found with the original algorithm.

We also performed a subgroup analysis by excluding women with a malignancy diagnosis (except nonmelanoma skin cancer) up to 5 years before study entry.

Statistical analyses were conducted using the statistical software package SAS version 9.2 (SAS Institute Inc, Cary, NC). The study was approved by the ethics committee of the Karolinska Institutet, Stockholm, Sweden.

\section{Results}

Using our algorithm, we identified 87 potential cases of ONJ among women with postmenopausal osteoporosis. Medical records were obtained for 83 of the cases $(95 \%)$. Fifteen out of these 83 potential cases were confirmed, yielding an overall PPV of $18 \%$ (95\% CI 10\%-28\%). Stratification 
on the selected ONJ-related diagnoses demonstrated that ICD-10 codes for periapical abscess with sinus (K04.6), alveolitis of jaws (K10.3), and other osteonecrosis (M87.8) had a PPV of 0 . It also revealed that codes on idiopathic aseptic necrosis of bone (M87.0), osteonecrosis due to previous trauma (M87.2), other secondary osteonecrosis (M87.3), and unspecified osteonecrosis (M87.9) were not used at all (Table 1).

Four patients had at separate occasions been recorded with two different diagnostic codes included in the algorithm (inflammatory conditions of jaws [K10.2] + osteonecrosis due to drugs [M87.1] or inflammatory conditions of jaws [K10.2] + other osteonecrosis [M87.8]). These four cases are presented in a mixed group category (Table 1). The stratification demonstrated that PPV was $100 \%$ when both inflammatory conditions of jaws and osteonecrosis due to drugs were recorded (K10.2+M87.1), whereas inflammatory conditions of jaws together with other osteonecrosis (K10.2+M87.8) had a PPV of 0. The highest PPV, mixed groups excluded, was for osteonecrosis due to drugs (M87.1) (83\%, 95\% CI 36\%-100\%).

In six potential cases, the second criterion (absence of healing after eight weeks despite adequate treatment) could not be confirmed (or ruled out), since the medical records did not cover the required eight weeks. These cases were thus not classified as ONJ.

The mean age among the 15 patients with confirmed ONJ was 74 years (standard deviation (SD) \pm 10 years), whereas the mean age among patients without confirmed
ONJ was 71 years ( $\mathrm{SD} \pm 8$ years). Six patients $(40 \%)$ had a prior malignancy diagnosis. Fourteen patients (93\%) had been treated with bisphosphonates and four patients $(27 \%)$ had been treated with cortisone. Three patients $(20 \%)$ had been treated with cytostatics (Table 2).

Dentoalveolar surgery had occurred prior to the start of the ONJ episode in eleven patients (73\%). In total, 20 ONJ lesions were described among the 15 confirmed cases. Eight lesions (40\%) were localized to the maxilla, whereas twelve lesions $(60 \%)$ occurred in the mandible (Table 2$)$.

Exclusion of women diagnosed with cancer up to five years before study entry reduced the PPV from $18 \%$ to $17 \%$ (95\% CI 9\%-28\%). When the diagnoses rendering a PPV of 0 were excluded, the overall PPV was $29 \%$. When we considered cases coded with inflammatory conditions of jaws (K10.2) as potential cases only if recorded at two or more visits to the department of oral and maxillofacial surgery, the overall PPV increased to $37 \%$ and the sensitivity decreased to $93 \%$. When requiring six months interval between the two visits, the overall PPV increased to $52 \%$, whereas the sensitivity decreased to $73 \%$.

\section{Discussion}

We evaluated an algorithm aimed at identifying osteonecrosis of the jaw (ONJ) using information recorded in the Swedish Patient Register. By medical record review we found that our algorithm had a positive predictive value (PPV) of $83 \%$ for osteonecrosis due to drugs (M87.1). Some diagnoses included in the algorithm had a PPV of 0 or were not used

Table I PPVs and 95\% Cls of diagnoses related to ONJ as recorded in the Swedish Patient Register and validated against medical records

\begin{tabular}{|c|c|c|c|c|}
\hline \multirow[t]{2}{*}{ ONJ related diagnoses in the patient register } & \multirow{2}{*}{$\begin{array}{l}\text { Potential cases } \\
\text { Number }\end{array}$} & \multicolumn{3}{|c|}{ Confirmed cases in the medical record review } \\
\hline & & Number & PPV $^{a}$ (\%) & $95 \% \mathrm{Cl}^{\mathrm{b}}$ \\
\hline Any ONJ related diagnosis & 83 & 15 & 18 & $10-28$ \\
\hline Periapical abscess with sinus (ICD-10 K04.6) & 20 & 0 & 0 & $0-17$ \\
\hline Inflammatory conditions of jaws (ICD-10 KI0.2) & 43 & 7 & 16 & $7-31$ \\
\hline Alveolitis of jaws (ICD-10 KI0.3) & 7 & 0 & 0 & $0-4 \mid$ \\
\hline Idiopathic aseptic necrosis of bone (ICD-I0 M87.0) & 0 & - & - & - \\
\hline Osteonecrosis due to drugs (ICD-10 M87.I) & 6 & 5 & 83 & $36-100$ \\
\hline Osteonecrosis due to previous trauma (ICD-I0 M87.2) & 0 & - & - & - \\
\hline Other secondary osteonecrosis (ICD-I0 M87.3) & 0 & - & - & - \\
\hline Other osteonecrosis (ICD-10 M87.8) & 3 & 0 & 0 & $0-7 \mid$ \\
\hline Osteonecrosis, unspecified (ICD-I0 M87.9) & 0 & - & - & - \\
\hline \multicolumn{5}{|l|}{ Mixed groups ${ }^{c}$} \\
\hline $\begin{array}{l}\text { Inflammatory conditions of jaws (ICD-10 KI0.2) + } \\
\text { osteonecrosis due to drugs (ICD-10 M87.I) }\end{array}$ & 3 & 3 & 100 & $29-100$ \\
\hline $\begin{array}{l}\text { Inflammatory conditions of jaws (ICD-10 KI0.2) + } \\
\text { other osteonecrosis (ICD-I0 M87.8) }\end{array}$ & 1 & 0 & 0 & $0-98$ \\
\hline
\end{tabular}

Notes: ${ }^{a}$ Number of confirmed cases divided by number of potential cases; ${ }^{b}$ confidence interval calculated using binomial exact method; ${ }^{c}$ potential cases with two different ICD-10 codes included in the algorithm.

Abbreviations: PPV, positive predictive value; $\mathrm{Cl}$, confidence interval; ONJ, osteonecrosis of the jaw; ICD, the International Classification of Diseases and Related Health Problems. 
Table 2 Characteristics of patients with a confirmed diagnosis of ONJ by medical record review (data from medical records)

\begin{tabular}{|c|c|}
\hline Characteristics & Number (\%) \\
\hline Total number of cases & 15 \\
\hline Mean age, years (SD) & $74(10)$ \\
\hline \multicolumn{2}{|l|}{ Age groups, years } \\
\hline $55-65$ & $3(20 \%)$ \\
\hline $66-75$ & $4(27 \%)$ \\
\hline$>75$ & $8(53 \%)$ \\
\hline \multicolumn{2}{|l|}{ Malignancies } \\
\hline \multicolumn{2}{|c|}{ (medical records and register data) } \\
\hline Breast cancer & $3(20 \%)$ \\
\hline Myeloma & I (7\%) \\
\hline Other & $2(13 \%)$ \\
\hline \multicolumn{2}{|l|}{ Comorbidities } \\
\hline Diabetes mellitus type 2 & $2(13 \%)$ \\
\hline Hypertension & $3(20 \%)$ \\
\hline Rheumatoid arthritis & I (7\%) \\
\hline Polymyalgia rheumatica & I (7\%) \\
\hline \multicolumn{2}{|l|}{ Medication } \\
\hline Bisphosphonates & 14 (93\%) \\
\hline Cytostatics & $3(20 \%)$ \\
\hline Glucocorticoids & $4(27 \%)$ \\
\hline Teriparatide & I (7\%) \\
\hline Proton-pump inhibitors & $2(13 \%)$ \\
\hline Dentures & $2(13 \%)$ \\
\hline Dentoalveolar surgery & II (73\%) \\
\hline Dental trauma & I (7\%) \\
\hline \multicolumn{2}{|l|}{ Localization } \\
\hline Right maxilla & $4(27 \%)$ \\
\hline Left maxilla & $4(27 \%)$ \\
\hline Left mandible & $6(40 \%)$ \\
\hline Right mandible & $6(40 \%)$ \\
\hline
\end{tabular}

Abbreviation: SD, standard deviation; ONJ, osteonecrosis of the jaw.

at all. When these diagnoses were excluded, the overall PPV of the algorithm increased from $18 \%$ to $29 \%$ without influencing the sensitivity.

The majority of patients suffering from ONJ (73\%) had been treated with some form of dentoalveolar surgery prior to the onset of ONJ. This corresponds well to what Filleul et $\mathrm{a}^{11}$ reported in a review of about $2400 \mathrm{ONJ}$ cases, where disease onset was preceded by tooth extraction in $67 \%$. Only $20 \%$ had no record of dentoalveolor surgery/trauma or dentures, which can be compared to the $26 \%$ that Filleul et $\mathrm{a}^{11}$ reported as spontaneous cases.

The majority of all ONJ lesions (60\%) occurred in the mandible, which is in line with other studies. Filleul et $\mathrm{al}^{11}$ reported that $65 \%$ of all $\mathrm{ONJ}$ lesions were localized to the mandible, whereas Hoff et al ${ }^{15}$ and Otto et $\mathrm{al}^{22}$ reported that $70 \%$ and $71 \%$ of all lesions, respectively, occur in the mandible. A total of $40 \%$ of the confirmed ONJ patients in our study had a prior malignancy diagnosis, and $27 \%$ had been receiving chemotherapy. Previous studies reported the highest occurrence of $\mathrm{ONJ}$ among patients who received intravenous bisphosphonates due to a malignant condition. ${ }^{23}$ Although this knowledge may cause a detection bias in our study, our subgroup analysis of women without cancer did not alter the results substantially.

A concern with this study is that it was conducted solely on osteoporotic women aged 55 years or older. Thus, the mean age of the ONJ patients in our study was 74 years old, which is higher than in several other studies. ${ }^{16,22,24}$ The mean age was 71 years among the potential ONJ cases that did not qualify for ONJ in the medical record review. Hence, our results support age as a risk factor in the development of ONJ. ${ }^{4}$ Furthermore, since osteoporosis was a selection criterion, it is not surprising that $93 \%$ of the patients had received bisphosphonates (intravenous or oral). Because there are no data available on the incidence of ONJ in the Swedish population, we could not determine the sensitivity, specificity, or negative predictive value of the evaluated algorithm, making us acknowledge another limitation of the present study.

Our algorithm includes several diagnostic codes on inflammation and osteonecrosis. They were selected based on clinical experience and hypothetical reasoning regarding which diagnostic codes to be used in coding ONJ, in the absence of a specific ICD-10 code for ONJ. Some of these codes are specific for the oral and maxillofacial region, whereas others are nonspecific for this site. Consequently, we combined these diagnostic codes with codes representing departments of oral and maxillofacial surgery, so that only conditions in the jaws were to be selected in the register search. Furthermore, whenever specified, conditions in anatomic sites other than the jaw were excluded. The time span of the study ran from 2005 through 2009. Since some required data in the Prescribed Drug Register was missing prior to 2005, this was the earliest possible study start.

Our case confirmation criteria are based on the definition proposed by AAOMS, which requires exposed bone in the mouth. Lately, however, reports on ONJ without exposed bone, so called nonexposed variant of ONJ, have been published. ${ }^{25}$ We chose not to include this variant in our case confirmation criteria for two reasons. Firstly, no definitive guideline for diagnosis of nonexposed variant of ONJ exists so far. ${ }^{25}$ Secondly, the nonexposed variant of ONJ was not known during the entire study period (2005 through 2009). Thus, clinicians that met patients with the nonexposed variant of ONJ during the study period, might not have considered it as ONJ. Consequently, inclusion of the nonexposed variant 
would have made case confirmation troublesome and might have led to false confirmation and overestimation of the PPV of the algorithm.

Our algorithm was developed for and validated using the Swedish Patient Register, which is a nationwide register comprising all physician visits. Thus, by utilizing the Patient Register, this algorithm has the potential to identify all ONJ cases in the entire country. Despite ONJ being an uncommon condition ${ }^{13}$ this also means that our algorithm has the potential to determine the incidence of ONJ in the general population, which currently is unknown. ${ }^{5,26}$ However, in Sweden, patients with ONJ are often taken care of by oral and maxillofacial surgeons or hospital dentists. Because only dentists in inpatient care are obligated to report to the Patient Register, the reporting from dentists in outpatient care might be incomplete.

The agreement between diagnostic codes in the Patient Register (other than those included in our algorithm) and medical records has been evaluated before. ${ }^{27} \mathrm{PPV}$ in most of these studies varied between $85 \%$ and $95 \%$, which has been considered sufficient to verify the register for the evaluated diagnostic code. Compared to these studies, the PPV of our algorithm was low. Our study, however, differs from these studies because ONJ does not have a specific ICD-10 code. Consequently, we did not validate an ICD-10 code, but we evaluated the performance of an algorithm ascertaining ONJ in the Patient Register.

Despite the difficulties conducting studies of ONJ in the Patient Register, an epidemiological approach may be one of the best, currently available strategies to learn more about this condition. In this study, we found that it is possible to identify cases of ONJ in the Patient Register using our algorithm. Although the PPV of the original algorithm was low, we have presented refinements that increase the PPV, with little effect on its sensitivity. By excluding some of the diagnoses in the original algorithm, and by sharpening the criteria for becoming a potential case among inflammatory conditions of jaws, the PPV was increased more than twofold. Thus, an algorithm including codes on inflammatory conditions of jaws (K10.2) and osteonecrosis due to drugs (M87.1) might prove to be a valuable instrument when conducting future register-based studies on ONJ.

\section{Disclosure}

The authors report no conflicts of interest in this work. The study was supported by unrestricted grants from Amgen. The funding source had no role in the conduct, analysis, or reporting of this study. None of the authors received any fees, honoraria, grants, or consultancies that would constitute a conflict of interest with the current study.

\section{References}

1. Marx RE. Pamidronate (Aredia) and zoledronate (Zometa) induced avascular necrosis of the jaws: a growing epidemic. J Oral Maxillofac Surg. 2003;61(9):1115-1117.

2. Hellstein JW, Marek CL. Bisphosphonate osteochemonecrosis (bis-phossy jaw): is this phossy jaw of the 21st century? J Oral Maxillofac Surg. 2005;63(5):682-689.

3. Silverman SL, Landesberg R. Osteonecrosis of the jaw and the role of bisphosphonates: a critical review. Am J Med. 2009;122(Suppl 2): S33-S45.

4. Ruggiero SL, Dodson TB, Assael LA, Landesberg R, Marx RE, Mehrotra B; for American Association of Oral and Maxillofacial Surgeons. American Association of Oral and Maxillofacial Surgeons position paper on bisphosphonate-related osteonecrosis of the jaws 2009 update. J Oral Maxillofac Surg. 2009;67(Suppl 5):2-12.

5. Khosla S, Burr D, Cauley J, et al; for American Society for Bone and Mineral Research. Bisphosphonate-associated osteonecrosis of the jaw: report of a task force of the American Society for Bone and Mineral Research. J Bone Miner Res. 2007;22(10):1479-1491.

6. Van Poznak C. Making a case for defining osteonecrosis of the jaw. J Dent Res. 2011;90(4):399-401.

7. Saad F, Brown JE, Van Poznak C, et al. Incidence, risk factors, and outcomes of osteonecrosis of the jaw: integrated analysis from three blinded active-controlled phase III trials in cancer patients with bone metastases. Ann Oncol. 2012;23(5):1341-1347.

8. Henry DH, Costa L, Goldwasser F, et al. Randomized, double-blind study of denosumab versus zoledronic acid in the treatment of bone metastases in patients with advanced cancer (excluding breast and prostate cancer) or multiple myeloma. J Clin Oncol. 2011;29(9):1125-1132.

9. Stopeck AT, Lipton A, Body JJ, et al. Denosumab compared with zoledronic acid for the treatment of bone metastases in patients with advanced breast cancer: a randomized, double-blind study. J Clin Oncol. 2010;28(35):5132-5139.

10. Fizazi K, Carducci M, Smith M, et al. Denosumab versus zoledronic acid for treatment of bone metastases in men with castrationresistant prostate cancer: a randomised, double-blind study. Lancet. 2011;377(9768):813-822.

11. Filleul O, Crompot E, Saussez S. Bisphosphonate-induced osteonecrosis of the jaw: a review of 2,400 patient cases. J Cancer Res Clin Oncol. 2010;136(8):1117-1124.

12. Migliorati CA, Siegel MA, Elting LS. Bisphosphonate-associated osteonecrosis: a long-term complication of bisphosphonate treatment. Lancet Oncol. 2006;7(6):508-514.

13. Ruggiero SL, Mehrotra B. Bisphosphonate-related osteonecrosis of the jaw: diagnosis, prevention, and management. Annu Rev Med. 2009;60:85-96.

14. Fleisher KE, Welch G, Kottal S, Craig RG, Saxena D, Glickman RS. Predicting risk for bisphosphonate-related osteonecrosis of the jaws: CTX versus radiographic markers. Oral Surg Oral Pathol Oral Radiol Endod. 2010;110(4):509-516.

15. Hoff AO, Toth B, Hu M, Hortobagyi GN, Gagel RF. Epidemiology and risk factors for osteonecrosis of the jaw in cancer patients. Ann NY Acad Sci. 2011;1218:47-54.

16. Vahtsevanos K, Kyrgidis A, Verrou E, et al. Longitudinal cohort study of risk factors in cancer patients of bisphosphonate-related osteonecrosis of the jaw. J Clin Oncol. 2009;27(32):5356-5362.

17. Landesberg R, Woo V, Cremers S, et al. Potential pathophysiological mechanisms in osteonecrosis of the jaw. Ann NY Acad Sci. 2011;1218: 62-79.

18. Rydh H. Patientregistret. [National Patient Register]. [webpage on the Internet]. Stockholm, Sweden: National Board of Health and Welfare. Available from: http://www.socialstyrelsen.se/register/halsodataregister/ patientregistret. Accessed March 27, 2012. Swedish. 
19. Rydh H. För uppgiftslämnare till patientregistret. [For information providers of the National patient Register]. [webpage on the Internet]. Stockholm, Sweden: National Board of Health and Welfare. Available from: http:/www.socialstyrelsen.se/register/halsodataregister/patientregistret/foruppgiftslamnarepatientregistret. Accessed March 27, 2012. Swedish.

20. Rydh H. Variabelförteckning. [Patientregistret. Record of variables the National Patient Register]. [webpage on the Internet]. Stockholm, Sweden: National Board of Health and Welfare. Available from: http:// www.socialstyrelsen.se/register/halsodataregister/patientregistret/ variabelforteckning. Accessed March 27, 2012. Swedish.

21. Leimanis A. Läkemedelsregistret. [Prescribed Drug Register]. [webpage on the Internet]. Stockholm, Sweden: National Board of Health and Welfare. Available from: http://www.socialstyrelsen.se/register/halsodataregister/lakemedelsregistret. Accessed March 27, 2012. Swedish.

22. Otto S, Schreyer C, Hafner S, et al. Bisphosphonate-related osteonecrosis of the jaws - characteristics, risk factors, clinical features, localization and impact on oncological treatment. J Craniomaxillofac Surg. 2012;40(4):303-309.
23. Reid IR, Cornish J. Epidemiology and pathogenesis of osteonecrosis of the jaw. Nat Rev Rheumatol. 2011;8(2):90-96.

24. Jung TI, Hoffmann F, Glaeske G, Felsenberg D. Disease-specific risk for an osteonecrosis of the jaw under bisphosphonate therapy. J Cancer Res Clin Oncol. 2010;136(3):363-370.

25. Patel S, Choyee S, Uyanne J, et al. Non-exposed bisphosphonaterelated osteonecrosis of the jaw: a critical assessment of current definition, staging, and treatment guidelines. Oral Dis. 2012;18(7): 625-632.

26. Yamashita J, McCauley LK, Van Poznak C. Updates on osteonecrosis of the jaw. Curr Opin Support Palliat Care. 2010;4(3):200-206.

27. Ludvigsson JF, Andersson E, Ekbom A, et al. External review and validation of the Swedish national inpatient register. BMC Public Health. 2011;11:450.
Clinical Epidemiology

\section{Publish your work in this journal}

Clinical Epidemiology is an international, peer-reviewed, open access journal focusing on disease and drug epidemiology, identification of risk factors and screening procedures to develop optimal preventative initiatives and programs. Specific topics include: diagnosis, prognosis treatment, screening, prevention, risk factor modification, systematic

Submit your manuscript here: http://www.dovepress.com/clinical-epidemiology-journal

\section{Dovepress}

reviews, risk \& safety of medical interventions, epidemiology \& biostatical methods, evaluation of guidelines, translational medicine, health policies \& economic evaluations. The manuscript management system is completely online and includes a very quick and fair peer-review system, which is all easy to use. 